\title{
High-density amorphous ice: A path-integral simulation
}

\author{
Carlos P. Herrero and Rafael Ramírez \\ Instituto de Ciencia de Materiales de Madrid, Consejo Superior de Investigaciones \\ Cientificas (CSIC), Campus de Cantoblanco, 28049 Madrid, Spain
}

(Dated: June 27, 2018)

\begin{abstract}
Structural and thermodynamic properties of high-density amorphous (HDA) ice have been studied by path-integral molecular dynamics simulations in the isothermal-isobaric ensemble. Interatomic interactions were modeled by using the effective $\mathrm{q}-\mathrm{TIP} 4 \mathrm{P} / \mathrm{F}$ potential for flexible water. Quantum nuclear motion is found to affect several observable properties of the amorphous solid. At low temperature $(T=50 \mathrm{~K})$ the molar volume of HDA ice is found to increase by $6 \%$, and the intramolecular $\mathrm{O}-\mathrm{H}$ distance rises by $1.4 \%$ due to quantum motion. Peaks in the radial distribution function of HDA ice are broadened respect to their classical expectancy. The bulk modulus, $B$, is found to rise linearly with the pressure, with a slope $\partial B / \partial P=7.1$. Our results are compared with those derived earlier from classical and path-integral simulations of HDA ice. We discuss similarities and discrepancies with those earlier simulations.

PACS numbers: 61.43.Er, 65.60.+a, 62.50.-p, 71.15.Pd
\end{abstract}

\section{INTRODUCTION}

Water is ubiquitous on Earth, and a detailed understanding of its properties and behavior under different conditions is of crucial importance in several scientific fields $\stackrel{\underline{1}-\underline{\underline{4}}}{\underline{\underline{a}}}$ This does not only refer to thermodynamically stable water phases, but also to various metastable phases obtained at ambient and extreme conditions of temperature and pressure. In particular, several forms of amorphous ice have been found and studied by both experimenta $\mathrm{l}^{-\underline{10}}$ and theoretical $\underline{11}^{114}$ methods, but some of their properties still lack a complete understanding. This is mainly due to the peculiar structure of condensed phases of water, where hydrogen bonds between adjacent molecules give rise to rather unusual properties of these phases.

The atomic dynamics in amorphous solids cause the appearance of localized low-energy excitations, displaying appreciable deviations from the situation of atomic nuclei harmonically vibrating around their potential

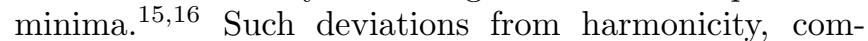
bined with the quantum character of atomic dynamics, is of great importance in the characterization of amorphous materials. In this context, the classical papers by Phillips $\frac{17}{}$ and Anderson et al $\stackrel{18}{\underline{10}}$ opened a research line by modeling low-energy excitations in amorphous solids by two-level systems. In more recent years there have appeared several detailed descriptions of the low-energy motion in this type of materials, beyond the standard tunneling model $\stackrel{19}{\underline{19}}$

When one studies average structural properties of amorphous materials, an interesting question is whether quantum effects can be noticeable in the presence of structural disorder. To be concrete, one may ask whether the radial distribution function (RDF) obtained in classical simulations is appreciably modified by considering quantum atomic delocalization, which can be important mainly at low temperatures. Two factors appear to compete in the broadening of the RDF peaks at low temper- ature: structural disorder and quantum delocalization $\underline{\underline{20}}$ As a first approach, one expects that for amorphous materials with heavy atoms (small zero-point vibrational amplitudes), structural disorder will broaden the peaks more than zero-point motion, and the opposite may occur for disordered materials with light atoms. For amorphous ice, one may suspect that the presence of hydrogen will make quantum effects appreciable, even for strong structural disorder.

Computer modeling of amorphous ice has been employed in recent years to obtain insight into its structural and dynamical properties $12,13,21-24$ The beginning of computer simulations of condensed phases of water at an atomic level dates back more than 40 years, $\stackrel{25,26}{ }$ and nowadays a large variety of empirical interatomic potentials can be found in the literature $27-33$ Many of them assume a rigid geometry for the water molecule, whereas some others allow for molecular flexibility either with harmonic or anharmonic $\mathrm{OH}$ stretches. In recent years, simulations of water using ab initio density functional theory (DFT) have been also carried out $34-36$ However, hydrogen bonds in condensed phases of water are difficult to describe with currently available energy functionals, making that some properties are not accurately reproduced by DFT calculations. $\stackrel{37}{ }$ Some progress in the description of van der Waals interactions in water within the DFT formalism has been recently made $\underline{38-41}$

A shortcoming of ab-initio electronic-structure calculations is that they usually deal with atomic nuclei as classical particles, disregarding quantum effects like zeropoint motion. These effects may be accounted for using harmonic or quasiharmonic approximations for the nuclear motion, but the precision of these approaches is not readily estimated when large anharmonicities are present, as can be the case for light atoms like hydrogen in disordered materials. To take into account the quantum character of atomic nuclei, the path-integral molecular dynamics (PIMD) approach has proved to be very useful, since in this procedure the nuclear degrees of freedom can 
be quantized in an efficient manner, thus including both quantum and thermal fluctuations in many-body systems at finite temperatures $\underline{42}$ This computational technique is now well established as a tool to study problems in which anharmonic effects can be important $\underline{43}$ Thus, a powerful approach could consist in combining DFT to determine the electronic structure and path integrals to describe the quantum motion of atomic nuclei $\sqrt[34.36]{36}$ However, this procedure requires computer resources that would restrict enormously the number of state points that can be considered in actual calculations.

Several forms of amorphous ice have been detected in recent years, corresponding to different densities $, 12,14,44-46$ In the present paper we study highdensity amorphous (HDA) ice by PIMD simulations at different pressures and temperatures, to analyze its structural and thermodynamic properties. Interatomic interactions are described by the flexible q-TIP4P/F model, which was recently developed and has been employed to carry out PIMD simulations of liquid $\underline{47} \underline{-49}$ and solid $\underline{50}-\underline{52}$ water. Here we pose the question of how quantum motion of the lightest atom can influence the structural properties of an amorphous water phase, and in particular if this quantum motion is appreciable for the solid at different densities, i.e. under different external pressures. This refers to the crystal volume and interatomic distances, but also to the mechanical stability of the solid. These questions have been addressed earlier for high- and low-

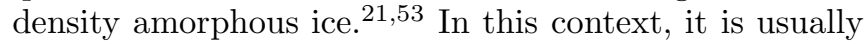
assumed that increasing quantum fluctuations enhances the exploration of the configuration space, but in certain regimes an increase in quantum fluctuations can lead to dynamical arrest, as found for glass formation. $\underline{\underline{5}}$

The paper is organized as follows. In Sec. II, we describe the computational method and the model employed in our calculations. In Sec. III we discuss the method employed to generate the simulation cells of amorphous ice. Our results are presented in Sec. IV, dealing with molar volume, interatomic distances, kinetic energy, and bulk modulus of HDA ice. Sec. V gives a comparison with earlier work, and Sec. VI includes a summary of the main results.

\section{COMPUTATIONAL METHOD}

We employ PIMD simulations to obtain several properties of amorphous ice at different temperatures and pressures. This kind of simulations are based on an isomorphism between a quantum system and a classical one, obtained after a discretization of the quantum density matrix along cyclic paths $\underset{5,56}{5}$ This isomorphism corresponds to replacing each quantum particle by a ring polymer consisting of $L$ (Trotter number) classical particles, joined by harmonic springs with temperature- and massdependent force constant. Details on this simulation procedure can be found elsewhere $\underline{\underline{42,43}}$ The dynamics used in this method is fictitious and does not correspond to the real quantum dynamics of the considered particles, but it helps to effectively sample the many-body configuration space, yielding precise results for the properties of the actual quantum system. Another way to derive such properties could be the use of Monte Carlo sampling, but we have found that this procedure requires for the present problem more computer resources than PIMD simulations. An important advantage of the latter is that the computing codes can be more readily parallelized, which turns out to be a relevant factor for an efficient use of modern computer architectures.

Simulations of crystalline and amorphous ice were carried out here in the isothermal-isobaric NPT ensemble ( $N$, number of particles; $P$, pressure; $T$, temperature), which allows one to find the equilibrium volume of a solid at given pressure and temperature. We have used effective algorithms for carrying out PIMD simulations in this statistical ensemble, similar to those described in the literature,,-579 and employed earlier in the study of solid and liquid water by PIMD simulations. We have considered temperatures between $50 \mathrm{~K}$ and $300 \mathrm{~K}$, and pressures up to $8 \mathrm{GPa}$. Both negative (tension) and positive (compression) pressures have been employed in the simulations. For negative $P$ we considered amorphous ice in the region of mechanical stability of the solid, down to $P \sim-0.5 \mathrm{GPa}$. For comparison with results of PIMD simulations, some simulations of HDA ice have been also performed in the classical limit, which is obtained in our path integral procedure by setting the Trotter number $L=1$.

Our PIMD simulations were carried out on cells including 96 or 216 water molecules, which were generated from pressure treatment of ice Ih and Ic supercells, respectively. The former (96 molecules) corresponds to a $(3 a, 2 \sqrt{3} a, 2 c)$ supercell, where $a$ and $c$ are the standard hexagonal lattice parameters of ice $\mathrm{Ih}$, whereas the latter (216 molecules) corresponds to a $3 \times 3 \times 3$ supercell of the cubic unit cell of ice Ic. The main purpose of taking these two ice types was to check the influence of the starting crystalline ice on the properties of the amorphous ice resulting under pressure. For all considered variables (structural and thermodynamic), we found in both cases results that coincided with each other within statistical error bars of the simulation procedure. To generate proton-disordered ice supercells (of Ih and Ic types) prior to amorphization, we employed a Monte Carlo procedure to impose that each oxygen atom had two chemically bonded and two H-bonded hydrogen atoms, and with a cell dipole moment close to zero $\stackrel{51.60}{5}$ For some particular conditions, we checked that both starting proton disorder and history of the amorphous supercell (amorphization procedure) do not affect significantly the results presented below. In particular, once formed the amorphous material from crystalline ice, we checked that its properties are reversible upon increasing and decreasing temperature and/or pressure.

Interatomic interactions were modeled by the point charge, flexible q-TIP4P/F model, developed to study 
liquid water, $\stackrel{47}{\longrightarrow}$ and that was later employed to study various properties of ice $\underline{50,51}$ and water clusters $\underline{61}$ Many of the empirical potentials used earlier for quantum simulations of condensed phases of water treat $\mathrm{H}_{2} \mathrm{O}$ molecules as rigid bodies $62-64$ This turns out to be convenient for computational efficiency, but neglects the role of intramolecular flexibility in the structure, dynamics, and thermodynamics of the condensed water phases $\stackrel{47}{ }$ Moreover, the q-TIP $4 \mathrm{P} / \mathrm{F}$ potential takes into account the significant anharmonicity of the $\mathrm{O}-\mathrm{H}$ vibration in a water molecule by considering anharmonic stretches, vs. the harmonic potentials employed in most of the simulations that considered quantum effects in these water phases.

Technical details on the PIMD simulations presented here are the same as those described and used in Refs. 50,51. The Trotter number $L$ has been taken proportional to the inverse temperature $(L \propto 1 / T)$, so that $L T=6000 \mathrm{~K}$, which allows us to keep roughly a constant precision in the PIMD results at the different temperatures under consideration. The time step $\Delta t$ employed for the calculation of interatomic forces in the molecular dynamics procedure was taken in the range between 0.1 and $0.3 \mathrm{fs}$, which was found to give adequate convergence for the variables studied here. For given conditions of pressure and temperature, a typical simulation run consisted of $2 \times 10^{5}$ PIMD steps for system equilibration, followed by $10^{6}$ steps for the calculation of ensemble average properties.

\section{PREPARATION OF AMORPHOUS ICE}

In this Section we give details on the form in which we obtain the disordered structures of HDA ice that are subsequently employed to characterize and study this amorphous phase from PIMD simulations. In particular, we have obtained simulation cells of HDA ice by applying a hydrostatic pressure to ice Ih and ice Ic at several temperatures. This procedure allows us also to check the pressure at which amorphization occurs, and to compare it with data (both experimental and theoretical) reported in the literature. We note that HDA ice has been recently obtained at room temperature from ice VII under rapid compression. 65

In Fig. 1 we present the pressure dependence of the molar volume of ice at a temperature of $75 \mathrm{~K}$, as derived from our PIMD simulations. Results shown as open circles correspond to simulations starting from ice Ih, for both negative and positive pressures. We observe that in the region between $-1 \mathrm{GPa}$ and $1 \mathrm{GPa}$ the volume decreases smoothly as pressure is increased, and at about 1.2 GPa it suffers a sudden decrease which corresponds to ice amorphization. This value is close to the spinodal pressure (limit of mechanical stability) obtained for ice Ih at this temperature in Ref. $67\left(P_{s}=1.19 \pm 0.05 \mathrm{GPa}\right)$, and to the amorphization pressure obtained in Ref. 22 from classical molecular dynamics simulations. From 1 to $2 \mathrm{GPa}$ the volume decreases by $27 \%$, and at higher

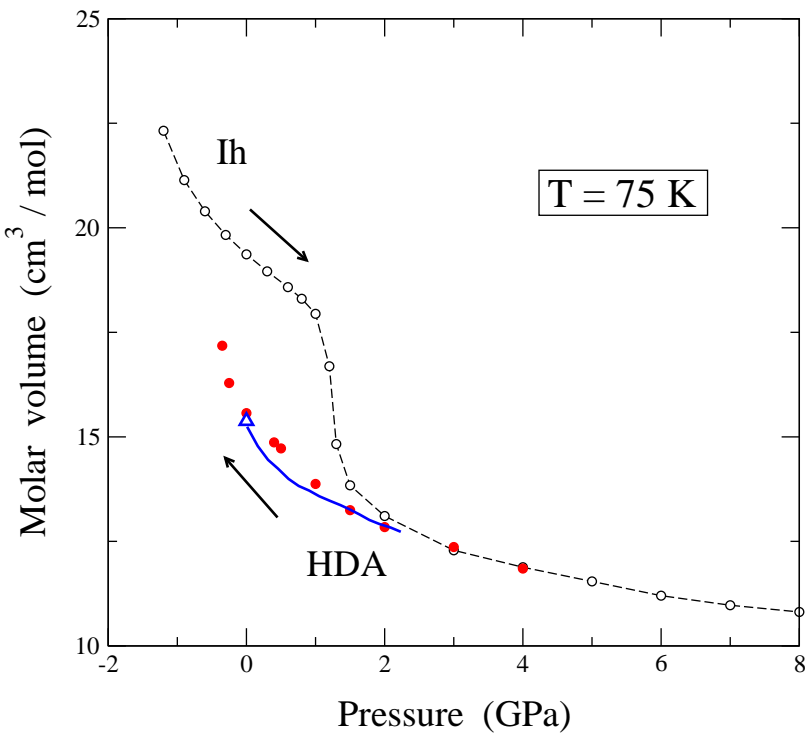

FIG. 1: Molar volume of ice as a function of pressure, as derived from PIMD simulations at $T=75 \mathrm{~K}$. Open circles represent results derived from simulations starting from ice Ih. Solid circles are data points obtained from simulations starting from amorphous ice. Error bars are in the order of the symbol size. The dashed line is a guide to the eye. An open triangle at $P=0$ indicates the volume measured by Mishima et $a l^{5}$. The solid line was obtained from the pressure-density data displayed in the review by Loerting and Giovambattista $^{14}$, taken from Ref. 66 .

pressures it continues decreasing to reach a value of 10.8 $\mathrm{cm}^{3} / \mathrm{mol}$ at $P=8 \mathrm{GPa}$, to be compared with $v=19.36$ $\mathrm{cm}^{3} / \mathrm{mol}$ found for ice Ih at atmospheric pressure.

We have carried out this procedure at several temperatures, and the results are similar to those shown in Fig. 1. In particular, the amorphization pressure changes slightly with temperature, and at $250 \mathrm{~K}$ we find 0.95 GPa. This was discussed in Ref. 67 in connection with the stability of ice Ih under pressure, and will not be repeated here. Apart from the pressure at which the solid amorphizes in the simulations, more precise values for the amorphization pressure can be obtained from the spinodal line of ice Ih, which gives the limit for the mechanical stability of this solid phase. This pressure corresponds to the vanishing of the bulk modulus (divergence of the compressibility), and can be approached in computer simulations at low temperatures $(T \lesssim 50 \mathrm{~K})$. At higher $T$ ice Ih amorphizes in the PIMD simulations before reaching the corresponding spinodal pressure, due to nucleation effects leading to the breakdown of the ice Ih structure $\underline{67}$ We note that the formation of HDA ice from ice Ih has not been observed in the laboratory at temperatures so high as $250 \mathrm{~K}$, but we obtain this transition at such temperatures and find an amorphous phase that remains metastable along our simulations (which in fact can only cover time scales shorter than those of actual experiments). HDA ice has been, however, obtained from 


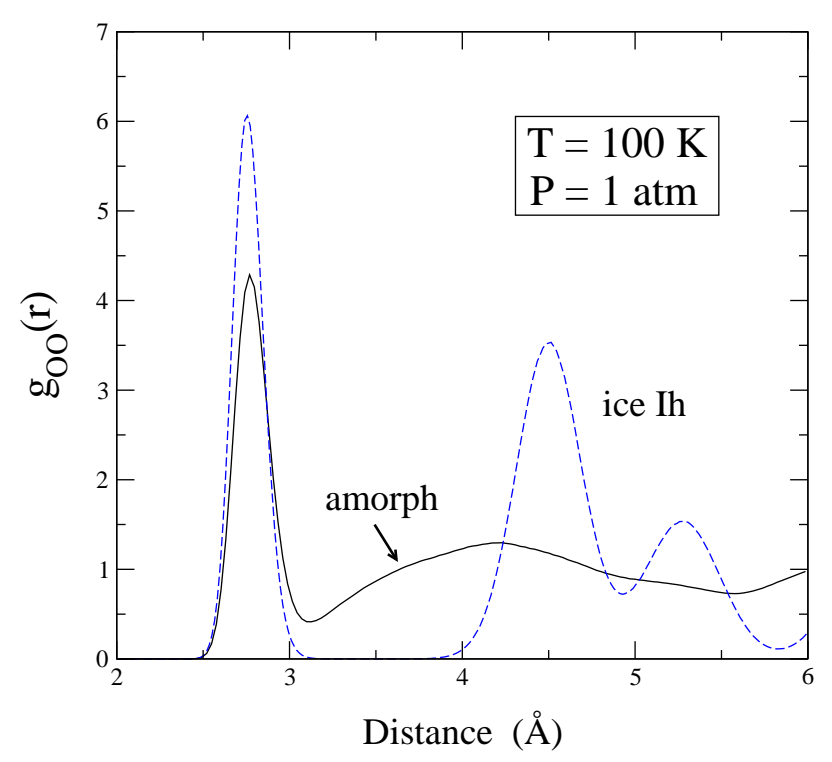

FIG. 2: Oxygen-oxygen radial distribution function of ice Ih (dashed line) and HDA ice (solid line) at $T=100 \mathrm{~K}$ and $P$ $=1 \mathrm{~atm}$, as derived from PIMD simulations.

ice VII at room temperature, $\underline{65}$ as mentioned above.

Solid water amorphizes in an irreversible way, so that new PIMD simulations at pressures lower than $1 \mathrm{GPa}$, starting from the amorphous phase, do not recover the crystalline phase. This is shown in Fig. 1 as solid symbols. In these simulations the pressure was gradually reduced down to negative pressures until reaching the limit of mechanical stability of the material. At $75 \mathrm{~K}$ we could reach a pressure of $-0.4 \mathrm{GPa}$, and at still more negative pressures the amorphous solid broke down along the simulations, transforming into the gas phase. This point will be discussed below in connection with the bulk modulus of HDA ice. For comparison with our results we present in Fig. 1 the molar volume obtained by Mishima et al $\underline{\underline{5}}^{\frac{5}{4}}$ for HDA ice from x-ray diffraction experiments at $80 \mathrm{~K}$ and atmospheric pressure. Shown is also the volumepressure curve derived from the data given in the review

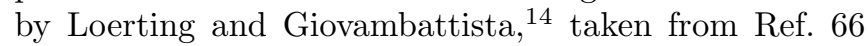
(solid line). We note that between $P=0$ and $1 \mathrm{GPa}$ the molar volume of the amorphous material is clearly smaller than that of the crystal, but at negative pressure the volume of the amorph increases fast, due to the proximity of its metastability limit, and therefore approaches the volume of ice Ih.

For additional confirmation of the amorphous character of the solid obtained after the cycle indicated by arrows in Fig. 1 (first pressure increase up to $8 \mathrm{GPa}$, and then pressure reduction), we display in Fig. 2 the $\mathrm{O}-\mathrm{O}$ RDF for ice Ih and amorphous ice, as derived from PIMD simulations at $T=100 \mathrm{~K}$ and atmospheric pressure. Upon amorphization, the prominent peaks corresponding to the second and third coordination sphere merge into one broad feature with a maximum at about 4.1 $\AA$. The maximum of the first peak in the amorphous solid remains close to that of ice Ih, in spite of the appreciable volume reduction suffered by the solid in the amorphization process. Note that the difference in height of the first peaks in the crystalline and amorphous phases is mainly due to the definition of radial distribution function, which is normalized by the density ${ }^{68}$ In fact, we calculate $g(r)$ as

$$
g(r)=\frac{d(r)}{D}
$$

where $D$ is the mean density (atoms per unit volume) and $d(r)$ is the "local density" at distance $r$ from a reference atom:

$$
d(r)=\frac{N(r)}{4 \pi r^{2} \Delta r},
$$

$N(r)$ being the number of atoms at distances between $r$ and $r+\Delta r$.

\section{PROPERTIES OF HIGH-DENSITY AMORPHOUS ICE}

\section{A. Volume}

As shown above in Fig. 1 and discussed in Sect. III, ice Ih suffers an important reduction in volume upon amorphization at about 1.2 GPa. This volume decrease is associated to a softening of intermolecular $\mathrm{O}-\mathrm{H}$ bridges, accompanied by a reduction in the mean distance to oxygen atoms in the second and third coordination shells (see Fig. 2). After amorphization, the molar volume continues decreasing as pressure is raised, and we find at 75 $\mathrm{K}$ a reduction from $13.11 \mathrm{~cm}^{3} / \mathrm{mol}$ at $P=2 \mathrm{GPa}$ to $10.81 \mathrm{~cm}^{3} / \mathrm{mol}$ at $8 \mathrm{GPa}$, which means a decrease of $18 \%$ respect to the volume at $2 \mathrm{GPa}$.

We emphasize that the volume of the amorph decreases smoothly in the whole pressure region considered here, and we did not detect in this region any other phase change as those reported in the literature. In fact, Hemley et $a l^{\underline{7}}$ observed a pressure-induced re-crystallization of HDA ice at about $4 \mathrm{GPa}$. Also, a slow transformation of HDA to cubic ice on slow depressurization has been observed $\stackrel{69}{\underline{6}}$ In our simulations the amorph remains in its metastable state in the whole range of temperature and pressure studied here.

In Fig. 3 we show the molar volume of ice Ih (circles) and the HDA phase (squares) as a function of temperature at atmospheric pressure, as derived from our PIMD simulations. At $P=1 \mathrm{~atm}$ and $T=75 \mathrm{~K}$, after releasing the pressure applied for amorphization, we find a molar volume $v=15.57 \mathrm{~cm}^{3} / \mathrm{mol}$, which corresponds to a density $\rho=1.16 \mathrm{~g} / \mathrm{cm}^{3}$, in good agreement with the values given by Mishima et al. at zero pressure: $\rho=$ $1.17 \mathrm{~g} / \mathrm{cm}^{3}$ in Ref. 5 and $1.19 \mathrm{~g} / \mathrm{cm}^{3}$ in Ref. 6 . For comparison with the PIMD results, we also display in Fig. 3 classical data derived here with the q-TIP4P/F potential 


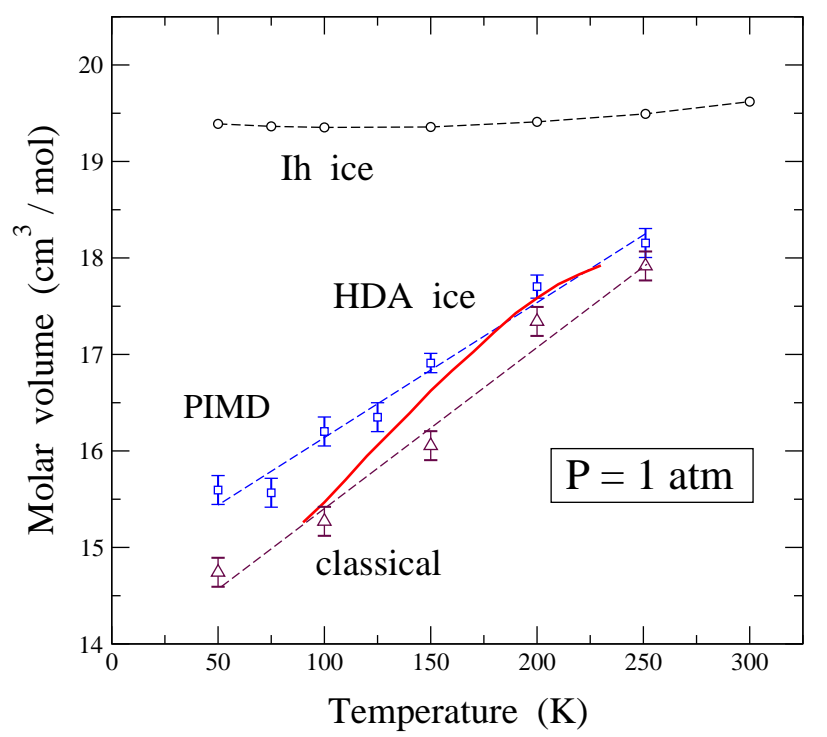

FIG. 3: Temperature dependence of the molar volume of ice at atmospheric pressure as derived from PIMD simulations: Ih (circles) and HDA ice (squares). Results of classical simulations for HDA ice are displayed as triangles. A solid line represents data obtained from classical molecular dynamics simulations by Tse et al.$^{13}$ For ice Ih the error bars are smaller than the symbol size. Lines are guides to the eye.

(triangles), as well as those obtained by Tse et al $\underline{\underline{13}}$ using the SPC/E potential. At low temperature, the molar volume found by these authors is similar to that found here in the classical simulations, and it becomes closer to the PIMD results as temperature rises. Comparing our results with the q-TIP4P/F force field, we find at $50 \mathrm{~K}$ an increase in molar volume of about $0.85 \mathrm{~cm}^{3} / \mathrm{mol}$ due to nuclear quantum effects, which amounts to a $6 \%$ of the classical value.

In the temperature region up to $250 \mathrm{~K}$ we did not observe any transition from HDA ice to a low-density amorphous phase with density $\rho=0.94 \mathrm{~g} / \mathrm{cm}^{3}$ (molar volume: $19.1 \mathrm{~cm}^{3} / \mathrm{mol}$ ), as experimentally observed at about 120 $130 \mathrm{~K}, 6,8,9,11$ and HDA ice remained as a metastable phase along our PIMD simulation runs. Something similar happens with the classical simulations reported in Ref. 13. We believe that such a transition between amorphous phases is not captured by the simulations due to the short time window that in fact can be observed in the calculations, similarly to the difficulties found in this kind of simulations to directly obtain transitions between different crystalline phases. This question could possibly be solved by performing direct coexistence simulations as those reported in the literature for liquid-solid transitions. $\underline{\underline{47}}$

It is clear that the amorphous material has a higher density, or a smaller molar volume than ice Ih, but it expands with temperature much faster than the crystalline solid. At atmospheric pressure we find for the amorph a volume increase of $2.6 \mathrm{~cm}^{3} / \mathrm{mol}$ in the temperature range

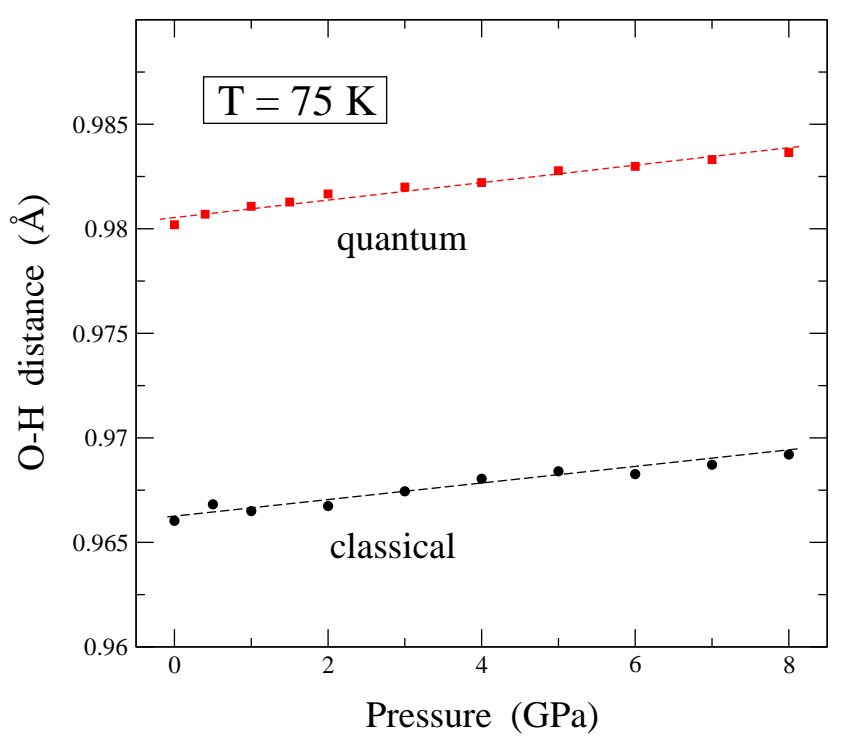

FIG. 4: Mean intramolecular $\mathrm{O}-\mathrm{H}$ distance as a function of pressure for amorphous ice at $75 \mathrm{~K}$, as derived from PIMD (squares) and classical (circles) simulations. Error bars are in the order of the symbol size. Lines are guides to the eye.

from 50 to $250 \mathrm{~K}$. In the same temperature region, the volume of ice Ih is found to rise less than $0.3 \mathrm{~cm}^{3} / \mathrm{mol}$, in part due to the negative thermal expansion around 100 $\mathrm{K}$ (not clearly observed at the scale of Fig. 3). Thus, we find for the thermal expansion coefficient of HDA ice at $100 \mathrm{~K}: \alpha=8.1 \times 10^{-4} \mathrm{~K}^{-1}$. This large volume expansion of the amorph, as compared with ice Ih, is indeed related with the lower bulk modulus of the amorphous material at $P=1 \mathrm{~atm}$ (see below).

\section{B. Interatomic distances}

In this section we present results for interatomic distances in amorphous ice between atoms in the same and adjacent molecules. This can shed light on the structural changes suffered by the material when temperature and/or pressure are modified. We first show in Fig. 4 the mean intramolecular $\mathrm{O}-\mathrm{H}$ distance as a function of pressure at a temperature $T=75 \mathrm{~K}$. In connection with this figure, there are two results that should be emphasized. First, at a given pressure, we find that the $\mathrm{O}-\mathrm{H}$ bond distance increases appreciably due to nuclear quantum effects. In fact, a classical simulation at $T=75 \mathrm{~K}$ and ambient pressure yields a mean $\mathrm{O}-\mathrm{H}$ distance of $0.966 \AA$, to be compared with $0.980 \AA$ derived from PIMD simulations, which means an increase of $1.4 \%$ in the bond length due to nuclear quantum motion. This difference is much larger than the temperature-induced change in $d(\mathrm{O}-\mathrm{H})$ at atmospheric pressure, which amounts to about 0.002 $\AA$ in the range from 50 to $250 \mathrm{~K}$. The increase due to quantum motion is rather constant in the whole pressure range studied here. 
Another important result observed in Fig. 4 is that at a given temperature the $\mathrm{O}-\mathrm{H}$ distance increases as pressure is raised, contrary to the usual contraction of atomic bonds for increasing $P$. This somewhat anomalous fact is due to the characteristic structure of ice with hydrogen bonds connecting contiguous water molecules, which gives rise to an anticorrelation between the strength of molecular $\mathrm{O}-\mathrm{H}$ bonds and intermolecular $\mathrm{H}$ bridges $.70 \mathrm{In}$ fact, increasing the pressure causes a hardening of intermolecular $\mathrm{H}$ bridges, with an associated weakening of intramolecular $\mathrm{O}-\mathrm{H}$ bonds, and a concomitant increase in the bond length. This weakening of intramolecular $\mathrm{O}-\mathrm{H}$ bonds in ice for rising pressure has been observed experimentally and reported in the literature. 71

It is interesting to compare the $\mathrm{O}-\mathrm{H}$ bond distance in amorphous ice with that obtained for ice Ih in the same type of PIMD simulations. At atmospheric pressure and $75 \mathrm{~K}$, we find for ice Ih a mean distance $d(\mathrm{O}-\mathrm{H})=0.984$ $\AA$, i.e. about $0.4 \%$ longer than in HDA ice at the same conditions 51 At the same temperature and $P=1 \mathrm{GPa}$, close to the amorphization pressure of ice Ih we found a distance difference of $0.6 \%$. This means that the average $\mathrm{O}-\mathrm{H}$ distance decreases upon amorphization of the solid, which is consistent with a weakening of $\mathrm{H}$ bridges between adjacent molecules, causing a strengthening of the intramolecular bonds, and therefore a shortening of the corresponding $\mathrm{O}-\mathrm{H}$ distance. These differences between $\mathrm{O}-\mathrm{H}$ distances in Ih and HDA ice are consistent with changes in the stretching vibrational frequencies, as observed from Raman scattering experiments. In fact, for HDA ice one observes at $80 \mathrm{~K}$ a broad Raman band with a maximum at $\approx 3200 \mathrm{~cm}^{-1}, 72$ to be compared with the largest feature appearing in the $\mathrm{O}-\mathrm{H}$ stretching region of ice Ih at about $3090 \mathrm{~cm}^{-1} .73$ This hardening of the stretching vibrations upon amorphization is consistent with the general trend found for water molecules, when the intramolecular $\mathrm{O}-\mathrm{H}$ distance decreases in different crystal surroundings: ${ }^{74} \Delta \omega(\mathrm{O}-\mathrm{H}) / \Delta d(\mathrm{O}-\mathrm{H})=-2.4 \times 10^{4}$ $\mathrm{cm}^{-1} / \AA$. We note, for comparison, that Bellissent-Funel et $a l^{75}$ found an intramolecular O-D distance of 0.97 $\AA$, from neutron scattering experiments on high-density amorphous $\mathrm{D}_{2} \mathrm{O}$.

Another related aspect of the $\mathrm{O}-\mathrm{H}$ distance is its temperature dependence. For ice Ih at atmospheric pressure, this distance is known to decrease as temperature is raised, as a consequence of the hardening of the bond for increasing temperature. This is in line with an enhancement of molecular motion for rising $T$, which causes a weakening of the $\mathrm{H}$ bridges and an associated enhancement of intramolecular bond strength. Something similar is found for amorphous ice in our PIMD simulations, where the average $\mathrm{O}-\mathrm{H}$ distance decreases from $0.9804(2)$ to $0.9788(2) \AA$ when $T$ rises from 75 to $250 \mathrm{~K}$.

Given that nuclear quantum motion is appreciable in the mean $\mathrm{O}-\mathrm{H}$ distance in water molecules in amorphous ice, it is expected that isotopic effects can be observed in the RDF. In Fig. 5 we display the $\mathrm{O}-\mathrm{H}$ radial distribution functions for HDA ice, as derived from PIMD simulations

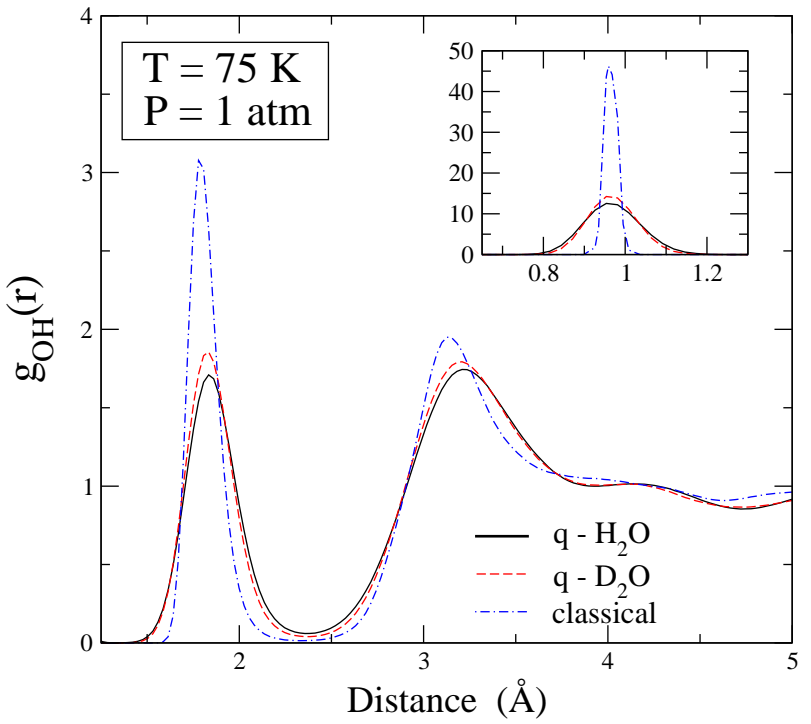

FIG. 5: Oxygen-hydrogen radial distribution function at 75 $\mathrm{K}$ and $P=1 \mathrm{~atm}$, as derived from quantum PIMD simulations for $\mathrm{H}_{2} \mathrm{O}$ (solid line) and $\mathrm{D}_{2} \mathrm{O}$ (dashed line) amorphous ice, as well as from classical molecular dynamics simulations (dashed-dotted line). Inset: $\mathrm{RDF}$ in the region around 1 $\AA$, showing the peak corresponding to intramolecular $\mathrm{O}-\mathrm{H}$ bonds.

for normal and deuterated water, as well as from classical molecular dynamics simulations. The RDF derived from classical simulations is similar to that found earlier in this kind of simulations for HDA ice 12 For interatomic distances $r$ from 1.5 to $5 \AA$, we observe in Fig. 5 that quantum effects cause a broadening of the peaks. This is particularly observable in the peaks at about $1.8 \AA$ and $3.2 \AA$. The first peak in this RDF, corresponding to the intramolecular $\mathrm{O}-\mathrm{H}$ bonds, is much higher, and is displayed in the inset. For the classical model, it is about 15 times larger than the peak at $1.8 \AA$. All peaks are found to broaden due to quantum effects, and their widths are larger for smaller isotopic mass. In fact, for the peak corresponding to intramolecular $\mathrm{O}-\mathrm{H}$ bonds, we obtained a full width at half maximum of $0.05,0.14$, and $0.16 \AA$, for classical ice, quantum $\mathrm{D}_{2} \mathrm{O}$, and quantum $\mathrm{H}_{2} \mathrm{O}$, respectively. Note that in this respect the classical limit behaves as the large-mass limit.

In Fig. 6 we show the hydrogen-hydrogen RDF of HDA ice as derived from classical (dashed line) and PIMD simulations (solid line). As expected, quantum motion of $\mathrm{H}$ broadens the peaks in the RDF with respect to the classical result. The peak corresponding to $\mathrm{H}-\mathrm{H}$ pairs inside water molecules, at $1.53 \AA$, has a height of 5.4 in the classical RDF, almost 4 times more than the quantum result of 1.45. In Fig. 6 we also present the $\mathrm{H}-\mathrm{H}$ RDF obtained by Finney et al. ${ }^{77}$ from neutron diffraction experiments at $80 \mathrm{~K}$. Note that this curve does not include the intramolecular $\mathrm{H}-\mathrm{H}$ pairs in the original publication. The next peak, corresponding to hydrogen pairs in ad- 


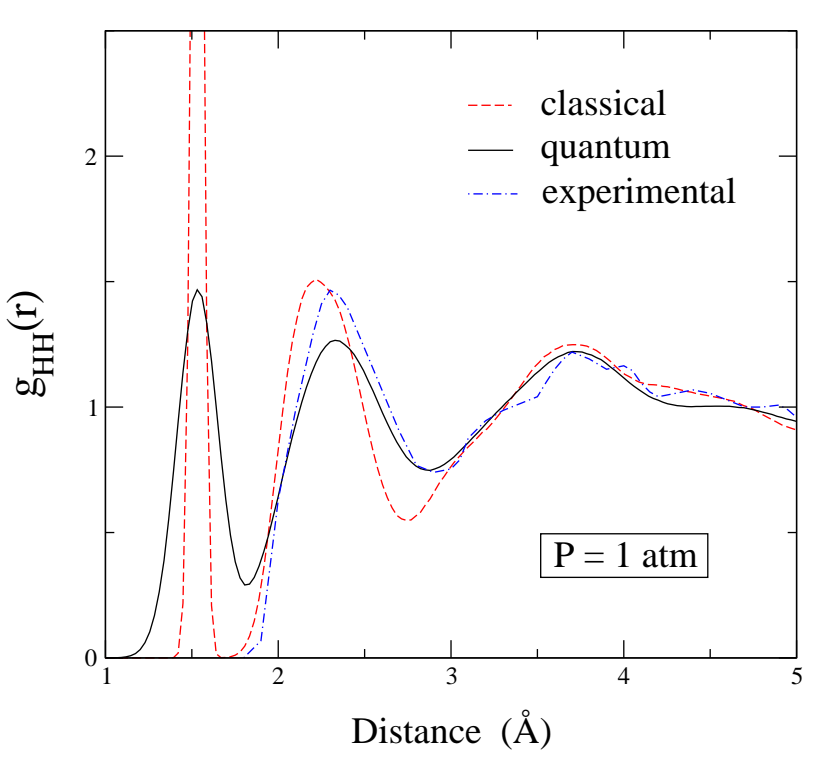

FIG. 6: Hydrogen-hydrogen radial distribution function for HDA ice at $P=1 \mathrm{~atm}$. Solid and dashed lines show results obtained from PIMD and classical simulations, respectively, at $T=75 \mathrm{~K}$. The dashed-dotted line was derived from neutron diffraction experiments at $80 \mathrm{~K}^{76}$

jacent (H-bonded) molecules appears nearly at the same position in the RDF derived from experiment and that obtained from PIMD simulations. However, the former is higher than the latter. This is the main difference between both results, that in general are rather similar.

Quantum effects are not only associated to hydrogen, due to its small mass, but are also observable in the oxygen-oxygen RDF of amorphous ice, as displayed in Fig. 7. In this figure, the solid line represents $g(r)$ derived from PIMD simulations at $T=75 \mathrm{~K}$ and atmospheric pressure, and the dashed line corresponds to the RDF derived from classical molecular dynamics simulations at the same conditions of pressure and temperature. The classical result is similar to the oxygen-oxygen RDF derived in Ref. 24 for several interatomic potentials. For comparison, we also show in Fig. 7 the $\mathrm{O}-\mathrm{O}$ RDF of HDA ice derived by Bowron et al. from neutron scattering experiments $\frac{45}{}$ (dashed-dotted line). As in the case of the $\mathrm{O}-\mathrm{H}$ RDF, we observe a broadening of the peaks when nuclear quantum motion is considered, as a consequence of the associated atomic delocalization. This is particularly observable for the first peak at about $2.8 \AA$, whose height decreases when quantum effects are taken into account. The result derived from PIMD simulations is closer to the experimental data than the classical one, but the peak derived from the quantum simulations appears at $r=2.77 \AA$, a distance slightly larger than that corresponding to the maximum of the RDF derived from experiment $(r=2.72 \AA)$. We note, however, that the position of the peak derived from another

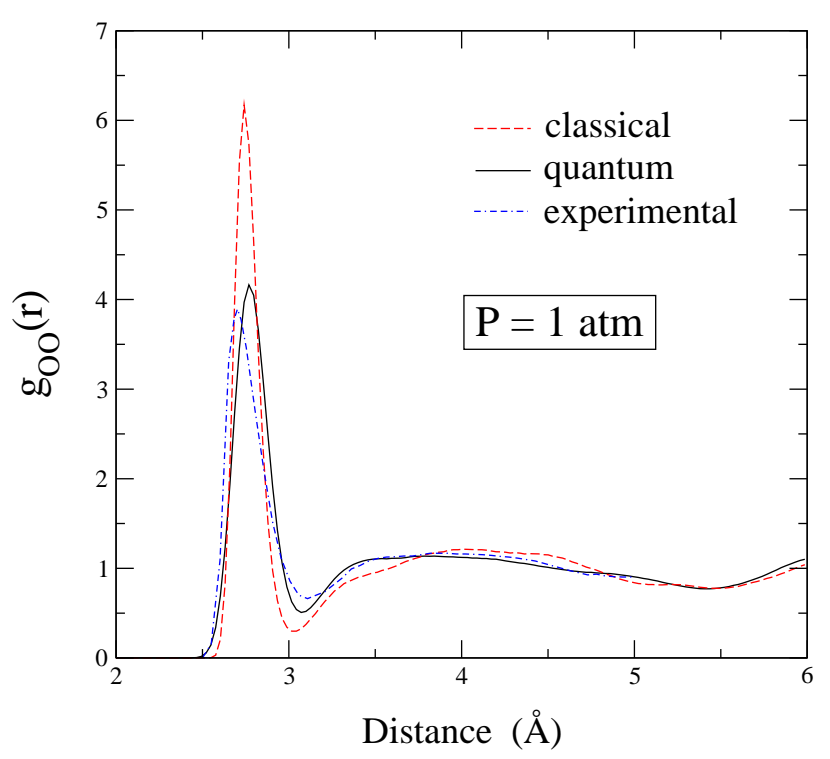

FIG. 7: Oxygen-oxygen radial distribution function for HDA ice at $P=1 \mathrm{~atm}$. Solid and dashed lines represent results derived from PIMD and classical simulations, respectively, at $T=75 \mathrm{~K}$. The dashed-dotted line was derived from neutronscattering experiments at $80 \mathrm{~K}^{45}$

neutron diffraction work ${ }^{77}$ is closer to that obtained in our simulations, but its height is somewhat smaller than that found here. RDFs of HDA ice have been also derived from x-ray diffraction measurements ${ }^{78}$

It is also interesting to analyze the effect of pressure on the shape of the $\mathrm{O}-\mathrm{O}$ RDF of amorphous ice, as it can give information on the molecular reorganization in the way to amorphous phases with still high density. With this purpose, in Fig. 8 we present the $\mathrm{O}-\mathrm{O}$ RDF at $75 \mathrm{~K}$ for different pressures: atmospheric pressure along with $P=1,3$, and $6 \mathrm{GPa}$. We first observe that the peak at about $2.8 \AA$, corresponding to the first coordination shell, moves to shorter distances as the pressure is raised, in line with a decrease in the $\mathrm{O}-\mathrm{O}$ distance associated to the corresponding volume reduction $(d V / d P<0)$. It is also remarkable that the broad feature appearing in the $\mathrm{RDF}$ around $4 \AA$ (at atmospheric pressure) sharpens and moves to smaller distances for increasing pressure, indicating that water molecules in the second coordination shell come closer to those in the first shell. This feature in the $\mathrm{O}-\mathrm{O} \mathrm{RDF}$ coincides with that observed in veryhigh density amorphous (VHDA) ice $\stackrel{14,76}{ }$ Other features of the RDF appearing at larger $r$ also move to shorter distances, as observed in the region between 5 and $6 \AA$ in Fig. 8. As shown above, we do not observe any clear discontinuity in the volume as pressure is increased, so that according to our results VHDA seems to appear as a high-pressure regime of HDA. Although in principle it is not evident that the phase obtained by applying pressure to HDA ice is the same as the VHDA obtained by isobaric heating $\stackrel{76}{\underline{76}}$ our results are in agreement with earlier simulations, $\underline{44}$ favoring a continuous transition from HDA 


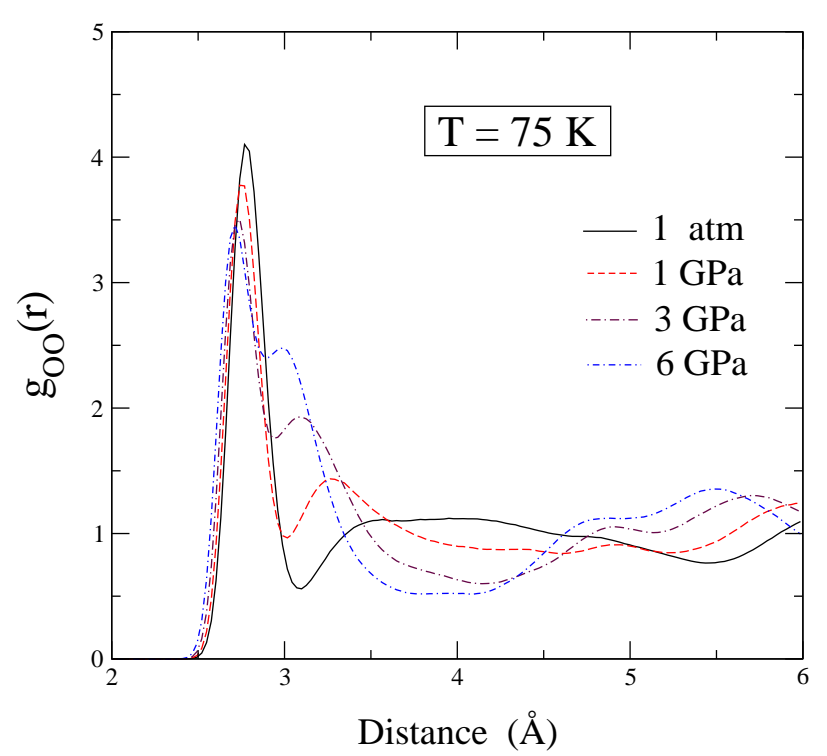

FIG. 8: Oxygen-oxygen radial distribution function for amorphous ice at $T=75 \mathrm{~K}$ and several pressures, as derived from PIMD simulations. Different lines represent results for $P=1 \mathrm{~atm}$, and 1, 3, $6 \mathrm{GPa}$, as indicated in the labels.

to VHDA ice. From an experimental point of view, there are indications $\frac{14}{}$ pointing in the direction that structural differences between HDA and VHDA become less prominent as pressure increases. In any case, there is some evidence against a first order-like nature of the transition between HDA and VHDA, suggesting a continuous character of the transition. $\underline{\underline{46}}$

\section{Kinetic energy}

In this section we present the kinetic energy of atomic nuclei in HDA ice, as derived from our PIMD simulations. The kinetic energy, $E_{k}$, of atomic nuclei depends on their mass and spacial delocalization, so that it can give information on the environment and interatomic interactions seen by the considered nuclei. We note that this does not occur for classical simulations, since in this case each degree of freedom contributes to the kinetic energy by an amount that depends only on temperature, i.e., $k_{B} T / 2$. A typical quantum effect associated to the atomic motion in solids is that the kinetic energy converges at low temperature to a value related to zero-point motion, contrary to the classical result where $E_{k}$ vanishes in the limit $T \rightarrow$ $0 \mathrm{~K}$. Path integral simulations allow us to obtain the kinetic energy of the considered quantum particles. For a particle with a certain mass at a given temperature, the larger the spread of the quantum paths, the smaller the kinetic energy, in line with the expectancy that a larger quantum delocalization is associated with a reduction in the kinetic energy. $\underline{42.51}$ We have calculated here the kinetic energy $E_{k}$ by using the so-called virial estimator, which has an associated statistical uncertainty apprecia-

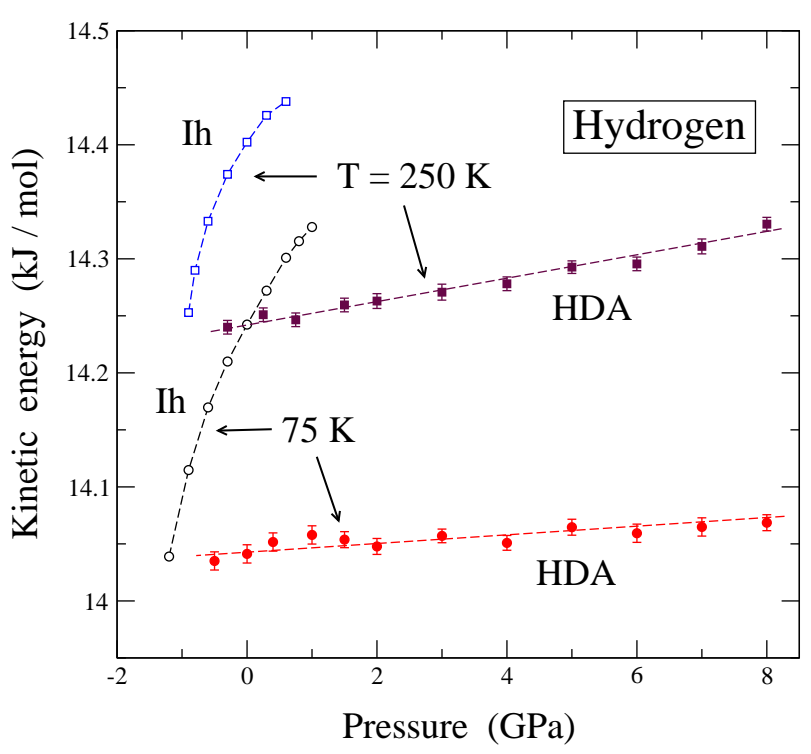

FIG. 9: Kinetic energy of hydrogen in ice Ih and amorphous ice as a function of pressure at two temperatures: 75 $\mathrm{K}$ (squares) and $250 \mathrm{~K}$ (circles). Open and solid symbols correspond to ice Ih and amorphous ice, respectively. Error bars for the crystalline phase are less than the symbol size. Lines are guides to the eye.

bly lower than the potential energy of the system. $\underline{58,79}$

In Fig. 9 we display $E_{k}$ for hydrogen as a function of pressure at two temperatures: 75 and $250 \mathrm{~K}$, as derived from our PIMD simulations of amorphous ice (solid symbols). At each temperature, $E_{k}$ increases slowly as pressure rises, corresponding to an overall increase of vibrational frequencies. For comparison, we also present results for hydrogen in ice Ih at the same temperatures (open symbols). In this case, the kinetic energy increases with rising pressure faster than for amorphous ice, in the whole region where ice Ih is found to be mechanically stable. This is a consequence of the larger quantum delocalization of hydrogen in amorphous ice, as compared with ice Ih at the same temperature, or equivalently, to the presence in the amorphous solid of modes with lower vibrational frequency. At atmospheric pressure, $E_{k}$ for HDA ice increases only by about $1.5 \%$ from 75 to 250 $\mathrm{K}$, reflecting the fact that at these temperatures most vibrational modes with large hydrogen contribution (i.e., libration and stretching modes) are nearly in their ground state. Moreover, changing the external pressure modifies even less the kinetic energy of hydrogen. In fact, at 75 $\mathrm{K}$ it rises by about $0.15 \%$ in the range from atmospheric pressure to $P=8 \mathrm{GPa}$.

In Fig. 10 we show the pressure dependence of the kinetic energy of oxygen in ice (HDA and Ih) at the same temperatures as those presented in Fig. 9 for hydrogen. We observe again that the kinetic energy rises with increasing pressure, and that at a given pressure, $E_{k}$ for oxygen in amorphous ice is smaller than in ice Ih. At 75 $\mathrm{K}$ and atmospheric pressure the kinetic energy of oxygen 


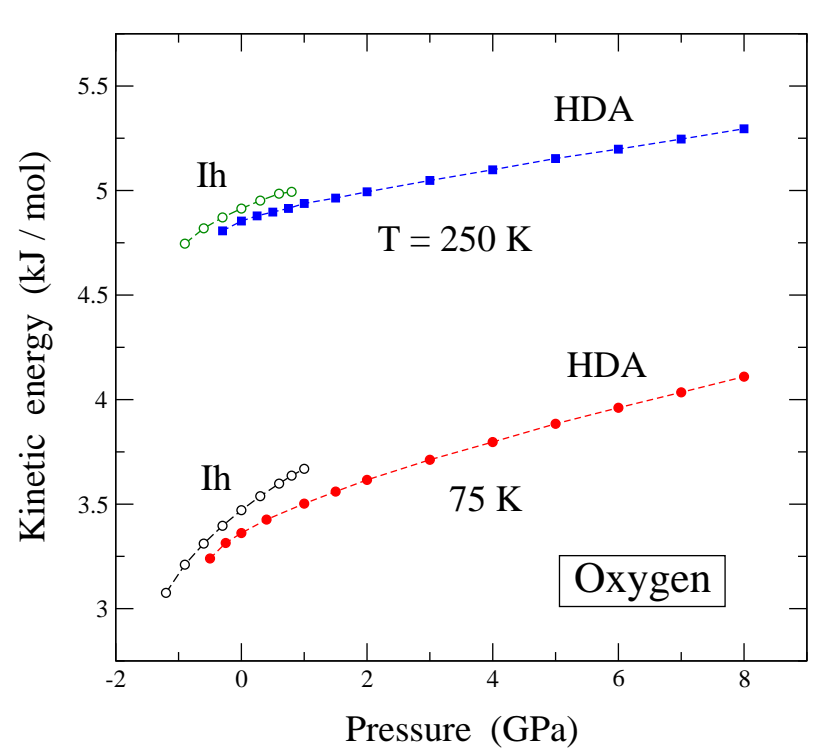

FIG. 10: Kinetic energy of oxygen in ice Ih and amorphous ice as a function of pressure at two temperatures: 75 $\mathrm{K}$ (squares) and $250 \mathrm{~K}$ (circles). Open and solid symbols correspond to ice Ih and amorphous ice, respectively. Error bars for the crystalline and amorphous solids are less than the symbol size. Lines are guides to the eye.

results to be about 4 times smaller than that of hydrogen, as could be expected from the larger mass of the former atom. At this temperature, $E_{k}$ for oxygen rises by about $22 \%$ when a pressure of $8 \mathrm{GPa}$ is applied. This relative change is much larger than that found for hydrogen at the same conditions (a $0.15 \%$ ). For increasing temperature, we find also an important rise in $E_{k}$ for oxygen, e.g., at $P=1 \mathrm{~atm}$ we have a change of a $44 \%$ from 75 to $150 \mathrm{~K}$, to be compared with a rise of $1.5 \%$ in the case of hydrogen. This is indeed a consequence of the much larger mass of oxygen, which contributes mainly to vibrational modes with low frequency, and therefore with excited states non-negligibly populated at these temperatures.

We note that the vertical scales in Figs. 9 and 10 are different, and what seems to be a very large change in the kinetic energy of hydrogen when comparing Ih and HDA ices, is in relative terms less than the change in $E_{k}$ for oxygen. In fact, at $P=1 \mathrm{GPa}$ and $T=75 \mathrm{~K}$ (close to the amorphization pressure of ice Ih), the kinetic energy of oxygen in HDA ice is $167 \mathrm{~J} / \mathrm{mol}$ lower than that in ice Ih, to be compared with a decrease of $270 \mathrm{~J} / \mathrm{mol}$ in $E_{k}$ for hydrogen. In relative terms these differences amount to a decrease of $4.5 \%$ for oxygen vs. $1.9 \%$ for hydrogen. This is in agreement with the fact that the structural changes associated to ice amorphization affect mainly to low-frequency vibrational modes (translational modes of the whole water molecule), with a major relative contribution of oxygen to the kinetic energy. The relative difference between kinetic energies in ice Ih and HDA ice decreases as temperature is raised, as observed in Figs. 9 and 10 at $T=250 \mathrm{~K}$, mainly because the nuclear motion becomes "more classical," and therefore $E_{k}$ is less sensitive to the environment and actual motion of the atomic nuclei under consideration.

The kinetic energy of hydrogen and oxygen in liquid water and ice Ih has been studied in detail earlier from PIMD simulations, and compared with data derived from deep inelastic neutron scattering in the case of hydrogen.$\underline{49}$ In that paper, a study of the contribution of different vibrational modes to the kinetic energy was presented. For HDA ice we find here that, at a given temperature, $E_{k}$ increases as pressure is raised for both hydrogen and oxygen. This result is not obvious, since the $\mathrm{O}-\mathrm{H}$ stretching frequencies are known to decrease for increasing pressure (their mode Grüneisen parameter is negative $\left.{ }^{41,80}\right)$. However, the overall contribution of the vibrational modes to $E_{k}$ increases with pressure, since the contribution of modes with positive Grüneisen parameter dominates, as analyzed elsewhere from a quasi-harmonic approximation for crystalline ice phases $\underline{\underline{80}}$

\section{Bulk modulus}

The compressibility of ice displays peculiar properties associated to the hydrogen-bond network. For the crystalline phases of ice, and ice Ih in particular, the compressibility is smaller than what one could suspect from the large cavities present in its structure, which could be expected to collapse under pressure before molecules could approach close enough to repel each other. This is in fact not the case, and for ice Ih the $\mathrm{H}$ bonds holding the structure are known to be rather stable, as manifested by the relatively high pressure necessary to break down the crystal. $\stackrel{5}{=}$ Here we present results of PIMD simulations for the isothermal bulk modulus of HDA ice at different temperatures and pressures, and compare them with those derived for ice Ih.

The isothermal compressibility $\kappa$ of ice, or its inverse the bulk modulus $\left[B=1 / \kappa=-V(\partial P / \partial V)_{T}\right]$ can be directly derived from PIMD simulations in the isothermal-isobaric ensemble. In this ensemble, $B$ can be obtained from the mean-square fluctuations of the volume, $\sigma_{V}^{2}=\left\langle V^{2}\right\rangle-\langle V\rangle^{2}$, by using the expression ${ }^{81.82}$

$$
B=\frac{k_{B} T\langle V\rangle}{\sigma_{V}^{2}}
$$

$k_{B}$ being Boltzmann's constant. This expression has been employed earlier to obtain the bulk modulus of different types of solids from path-integral simulations $.51,82,83$

In Fig. 11 we present the bulk modulus of amorphous ice as a function of pressure, as derived from our PIMD simulations at $75 \mathrm{~K}$ (solid circles) and $250 \mathrm{~K}$ (solid squares). For comparison, we also present results for ice $\mathrm{Ih}$ at $75 \mathrm{~K}$ (open circles). The bulk modulus of amorphous ice is found to increase linearly as a function of pressure in the range considered here. From linear fits to 


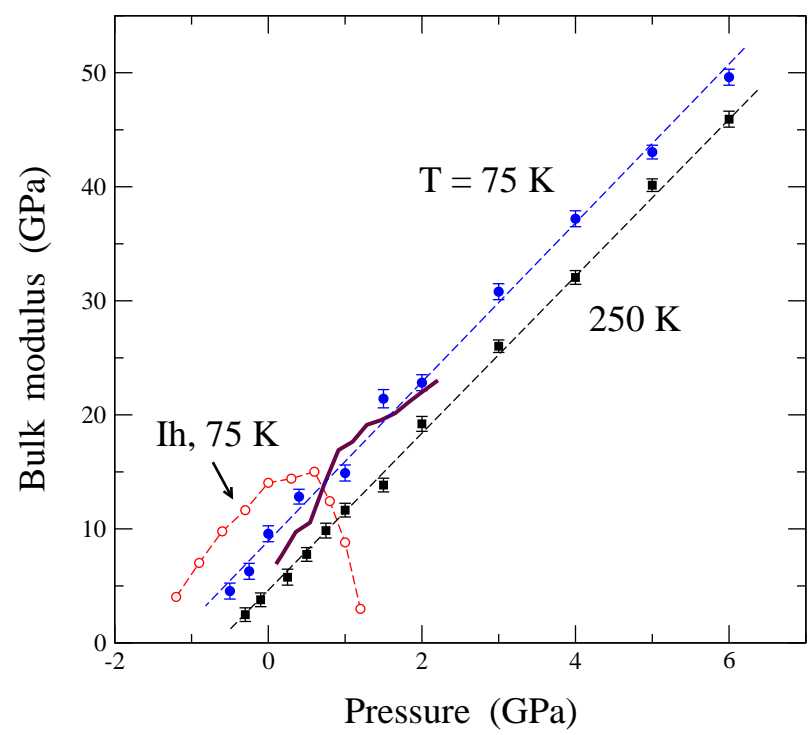

FIG. 11: Pressure dependence of the bulk modulus of amorphous ice at $T=75 \mathrm{~K}$ (solid circles) and $250 \mathrm{~K}$ (solid squares), as derived from PIMD simulations. Open circles correspond to ice Ih at $75 \mathrm{~K}$. Error bars for the crystalline phase are in the order of the symbol size. Dashed lines are guides to the eye. The solid line was obtained by numerical differentiation from the pressure-density data displayed in the review by Loerting and Giovambattista, ${ }^{14}$ adapted from Ref. 66 .

the data shown for amorphous ice in Fig. 11, we find a slope $\partial B / \partial P=7.1(2)$ and $7.0(2)$ at 75 and $250 \mathrm{~K}$, respectively, i.e. both values coincide within the precision of our results. This contrasts clearly with the pressure dependence of the bulk modulus found for ice Ih (open circles in Fig. 11). In fact, for this crystalline phase of water one finds that at $P>0.3 \mathrm{GPa}, B$ decreases as pressure is raised, until eventually reaching the limit of mechanical stability of the phase (spinodal line) and the associated amorphization of the material. Thus, at pressures close to $1 \mathrm{GPa}$, the bulk modulus of ice Ih is lower than that of the amorphous phase, contrary to the result obtained at lower pressures. For comparison, we also show in Fig. 11 the pressure dependence of the bulk modulus of HDA ice at $80 \mathrm{~K}$ (solid line), derived by numerical differentiation of the pressure-density data given in Ref. 14 (adapted from 66).

At atmospheric pressure and $75 \mathrm{~K}$, we find for amorphous ice $B=9.6(4) \mathrm{GPa}$, to be compared with a value of $B=14.0(3) \mathrm{GPa}$ derived for ice Ih at the same conditions. This reflects an important increase in the compressibility of ice upon amorphization, i.e. a lowering of the bulk modulus of the material by about a $30 \%$. Note that this reduction in the bulk modulus is similar to the relative volume change upon amorphization, as discussed in Sect. III.

From our data in the temperature region between 75 and $250 \mathrm{~K}, B$ extrapolates to zero at negative pressures in the order of -0.5 to $-1 \mathrm{GPa}$, which gives the limit of me- chanical stability of this amorphous material, where the solid breaks down giving rise to the gas phase. As discussed elsewhere, $, 67,84$ the possibility of studying a solid in metastable conditions, close to a spinodal line is limited by the appearance of nucleation events, which cause the breakdown of the solid. For atomistic simulations such as those employed here, the probability of those nucleation events at low temperatures is relatively low, and the metastable range of the solid that can be explored is rather large. In fact, we can go here to conditions near the limit of mechanical stability at negative pressures (limit $B \rightarrow 0$ ). As $T$ increases the probability of nucleation becomes higher, and the accessible pressure range in the simulations is reduced.

\section{COMPARISON WITH EARLIER WORK}

Data of earlier simulations of HDA ice have been already presented in the previous section along with the results of our PIMD simulations. Here we discuss and summarize the main similarities and discrepancies between our results and those given in some earlier works.

Simulations of the amorphization of ice Ih using classical molecular dynamics were carried out by Tse and Klein, $, 85,86$ who employed the TIP4P intermolecular potential, and found ice amorphization at pressures around 1.2-1.3 GPa at temperatures between 80 and $100 \mathrm{~K}$. These values are close to the limit for mechanical stability (spinodal pressure, $P_{s}$ ) of ice Ih obtained from PIMD simulations, i.e., $P_{s}=1.12$ and $1.26 \mathrm{GPa}$ at 50 and 100 $\mathrm{K}$, respectively. 67

Seidl et $a l^{24}$ carried out detailed classical molecular dynamics simulations of HDA ice in the isothermalisobaric ensemble using several force fields. In particular, they studied the glass-transition at a pressure of $0.3 \mathrm{GPa}$, and found some indications of a glass-to-liquid transition at a temperature around $200 \mathrm{~K}$, which could suggest that HDA ice is a proxy of an ultraviscous high-density liquid. From our present results, we cannot find any evidence of such a transition, and a detailed study of this point with PIMD simulations would require at present enormous computational resources.

In connection with our work, Tse et al. $\stackrel{13}{s}$ studied amorphous ice by classical molecular dynamics simulations with the SPC/E potential. They presented O-O RDFs very similar to those obtained in our classical simulations (shown in Fig. 7). These authors found a continuous and smooth increase in the molar volume of HDA ice as temperature is raised, without a sharp change indicating transformation from high-density to low-density amorphous phases. This is in line with the results of our PIMD simulations shown in Fig. 3. In particular, they found at $100 \mathrm{~K}$ a molar volume of $15.4 \mathrm{~cm}^{3} / \mathrm{mol}$, close to our classical result (triangles in Fig. 3) and smaller than the value derived from our PIMD simulations with the $\mathrm{q}$-TIP $4 \mathrm{P} / \mathrm{F}$ potential $\left(v=16.2 \mathrm{~cm}^{3} / \mathrm{mol}\right)$. At higher temperatures the results by Tse et al. become closer to 
our PIMD results, and are therefore somewhat higher than our classical data. This can be a consequence of the differences between the force fields employed in both works.

Earlier studies of path integral simulations of amorphous ice are scarce. Gai et $a l^{21}$ studied structural properties of HDA ice at $77 \mathrm{~K}$ by path-integral Monte Carlo simulations, using the SPC/E potential model, which treats the water molecules as rigid bodies. Their simulations were carried out in the constant volume, canonical $(N V T)$ ensemble, and dealt with both $\mathrm{H}_{2} \mathrm{O}$ and $\mathrm{D}_{2} \mathrm{O}$ ice. The $\mathrm{O}-\mathrm{H}$ RDF obtained by these authors for $\mathrm{D}_{2} \mathrm{O}$ amorphous ice is similar to that obtained here, and presented in Fig. 5. In particular, they found well-defined peaks for the first and second coordination shells at about 1.8 and $3.3 \AA$, respectively. However, for $\mathrm{H}_{2} \mathrm{O}$ ice they found a RDF very different to that found here, in which the peaks at $1.8 \AA$ (intermolecular $\mathrm{O}-\mathrm{H}$ bridges) and $3.3 \AA$ were missing, and had been replaced by a broad feature extending from about 2.5 to $4 \AA$. These authors argued that the hydrogen bonding network that is present for $\mathrm{D}_{2} \mathrm{O}$ either disappears or is totally mixed with the second nearest-neighbor shell.

Apart from the constant volume employed by Gai et $a l .21$ in their simulations vs the constant pressure employed in ours, the main difference between both kinds of calculations seems to be the interatomic potential: rigid molecules in Ref. 21 vs flexible molecules in our calculations. We do not find, however, a direct explanation why the use of rigid molecules should cause a so strong difference between the structures of $\mathrm{H}_{2} \mathrm{O}$ and $\mathrm{D}_{2} \mathrm{O}$ amorphous ice, as suggested by the results of Gai et al.

\section{SUMMARY}

PIMD simulations provide us with a suitable tool to analyze effects of nuclear quantum motion in amorphous ice at finite temperatures. Here, we have presented results of PIMD simulations of HDA ice in the isothermalisobaric ensemble at different pressures and temperatures. This kind of simulations have allowed us to obtain structural and thermodynamic properties of this metastable material in a large region of pressures, including tensile stresses $(P<0)$.

The HDA ice studied here was obtained computationally by pressure-induced amorphization of cubic and hexagonal ice (Ih and Ic). We observe an important reduction of the volume upon amorphization at a pressure of about 1.2 GPa. The resulting ice at atmospheric pressure and $100 \mathrm{~K}$ is $19 \%$ denser than its crystalline precursor, but it is found to be softer, in the sense that its compressibility and thermal expansion coefficient are clearly larger. At $P=1$ atm and $T=75 \mathrm{~K}$, the compressibility $\kappa$ of HDA ice is about $50 \%$ larger than that of ice Ih, and the thermal expansion coefficient $\alpha$ for the amorphous solid is found to be $9 \times 10^{-4} \mathrm{~K}^{-1}$, whereas it is negative for ice Ih at $75 \mathrm{~K}$.

We have assessed the importance of quantum effects by comparing results obtained from PIMD simulations with those obtained from classical simulations. Structural variables are found to change when nuclear quantum motion is considered, especially at low temperatures. Thus, the crystal volume, interatomic distances, and radial distribution functions suffer appreciable modifications in the range of temperature and pressure considered here. At $50 \mathrm{~K}$ the molar volume of HDA ice is found to rise by $0.85 \mathrm{~cm}^{3} / \mathrm{mol}$ (a $6 \%$ of the classical value), and the intramolecular $\mathrm{O}-\mathrm{H}$ distance increases by $1.4 \%$ due to quantum motion.

The zero-point vibrational motion of atomic nuclei is large enough to also change appreciably structural observables of the amorphous solid, such as the radial distribution function at low temperatures. In fact, from PIMD simulations we observe a broadening of the peaks in the RDFs, as compared with classical molecular dynamics simulations. For different isotopes we also observe a change in the RDFs of HDA ice. In particular, the width of the peaks in the $\mathrm{O}-\mathrm{H}$ RDF is found to depend on the hydrogen isotope under consideration, but the general features of this RDF are basically the same for both $\mathrm{H}_{2} \mathrm{O}$ and $\mathrm{D}_{2} \mathrm{O}$, contrary to earlier path-integral Monte Carlo results. 21

At a given temperature, the kinetic energy of both hydrogen and oxygen is found to increase for rising pressure. This increase is, however, slower than that obtained in ice Ih. Such an increase is associated to the rise in vibrational zero-point energy and an overall increase in the vibrational frequencies of the solid. However, the intramolecular $\mathrm{O}-\mathrm{H}$ distance is found to increase as pressure is raised, with a decrease in the frequency of the corresponding stretching modes. In HDA ice the bulk modulus is found to increase linearly as a function of pressure, in the whole region studied here. At $75 \mathrm{~K}$ we find $\partial B / \partial P=7.1$.

Although quantitative values found by using the q$\mathrm{TIP} 4 \mathrm{P} / \mathrm{F}$ potential can change by employing other interatomic potentials, the main conclusions obtained here can hardly depend on the potential employed in the simulations. Quantum simulations similar to those presented here can give information on the atom delocalization and anharmonic effects in other kinds of amorphous ice. An extension of this work could consist in studying amorphous ice at still higher pressures, where very-high density amorphous ice could be characterized, including nuclear quantum effects.

\section{Acknowledgments}

This work was supported by Ministerio de Ciencia e Innovación (Spain) through Grant FIS2009-12721-C04-04 and by Comunidad Autónoma de Madrid through Program MODELICO-CM/S2009ESP-1691. 
1 D. Eisenberg and W. Kauzmann, The Structure and Properties of Water (Oxford University Press, New York, 1969).

${ }^{2}$ V. F. Petrenko and R. W. Whitworth, Physics of Ice (Oxford University Press, New York, 1999).

${ }^{3}$ F. Franks, Water: A Matrix of Life (Royal Society of Chemistry, London, 2000), 2nd ed.

${ }^{4}$ G. W. Robinson, S. B. Zhu, S. Singh, and M. W. Evans, Water in Biology, Chemistry and Physics (World Scientific, Singapore, 1996).

5 O. Mishima, L. D. Calvert, and E. Whalley, Nature 310, 393 (1984).

6 O. Mishima, L. D. Calvert, and E. Whalley, Nature 314, 76 (1985).

7 R. J. Hemley, L. C. Chen, and H. K. Mao, Nature 338, 638 (1989).

8 C. A. Tulk, C. J. Benmore, J. Urquidi, D. D. Klug, J. Neuefeind, B. Tomberli, and P. A. Egelstaff, Science 297, 1320 (2002).

9 R. J. Nelmes, J. S. Loveday, T. Strässle, C. L. Bull, M. Guthrie, G. Hamel, and S. Klotz, Nature Phys. 2, 414 (2006).

10 T. Strässle, S. Klotz, G. Hamel, M. M. Koza, and H. Schober, Phys. Rev. Lett. 99, 175501 (2007).

11 J. S. Tse, D. D. Klug, C. A. Tulk, I. Swainson, E. C. Svensson, C. K. Loong, V. Shpakov, V. R. Belosludov, R. V. Belosludov, and Y. Kawazoe, Nature 400, 647 (1999).

12 N. Giovambattista, H. E. Stanley, and F. Sciortino, Phys. Rev. E 72, 031510 (2005).

13 J. S. Tse, D. D. Klug, M. Guthrie, C. A. Tulk, C. J. Benmore, and J. Urquidi, Phys. Rev. B 71, 214107 (2005).

14 T. Loerting and N. Giovambattista, J. Phys.: Condens. Matter 18, R919 (2006).

15 N. E. Cusack, The Physics of Structurally Disordered Matter (Adam Hilger, Bristol, 1987).

16 S. R. Elliott, Physics of Amorphous Materials (Longman, New York, 1990).

17 W. A. Phillips, J. Low Temp. Phys. 7, 351 (1972).

18 P. W. Anderson, B. I. Halperin, and C. M. Varma, Phil. Mag. 25, 1 (1972).

19 M. A. Ramos and U. Buchenau, in Tunneling Systems in Amorphous and Crystalline Solids, edited by P. Esquinazi (Springer, Berlin, 1998), p. 527.

20 C. P. Herrero, Europhys. Lett. 44, 734 (1998).

${ }^{21}$ H. Gai, G. K. Schenter, and B. C. Garrett, Phys. Rev. B 54, 14873 (1996).

22 I. Okabe, H. Tanaka, and K. Nakanishi, Phys. Rev. E 53, 2638 (1996).

23 N. Giovambattista, C. A. Angell, F. Sciortino, and H. E. Stanley, Phys. Rev. Lett. 93, 047801 (2004).

24 M. Seidl, T. Loerting, and G. Zifferer, J. Chem. Phys. 131, 114502 (2009).

25 J. A. Barker and R. O. Watts, Chem. Phys. Lett. 3, 144 (1969).

26 A. Rahman and F. H. Stillinger, J. Chem. Phys. 55, 3336 (1971).

27 M. W. Mahoney and W. L. Jorgensen, J. Chem. Phys. 115, 10758 (2001).

28 Y. Koyama, H. Tanaka, G. Gao, and X. C. Zeng, J. Chem. Phys. 121, 7926 (2004).

${ }^{29}$ W. L. Jorgensen and J. Tirado-Rives, PNAS 102, 6665
(2005)

30 J. L. F. Abascal and C. Vega, J. Chem. Phys. 123, 234505 (2005).

31 F. Paesani, W. Zhang, D. A. Case, T. E. Cheatham, and G. A. Voth, J. Chem. Phys. 125, 184507 (2006).

32 C. McBride, C. Vega, E. G. Noya, R. Ramírez, and L. M. Sesé, J. Chem. Phys. 131, 024506 (2009).

33 M. A. Gonzalez and J. L. F. Abascal, J. Chem. Phys. 135, 224516 (2011).

34 B. Chen, I. Ivanov, M. L. Klein, and M. Parrinello, Phys. Rev. Lett. 91, 215503 (2003).

35 M. V. Fernández-Serra and E. Artacho, Phys. Rev. Lett. 96, 016404 (2006).

36 J. A. Morrone and R. Car, Phys. Rev. Lett. 101, 017801 (2008).

37 S. Yoo, X. C. Zeng, and S. S. Xantheas, J. Chem. Phys. 130, 221102 (2009).

38 J. Wang, G. Roman-Perez, J. M. Soler, E. Artacho, and M. V. Fernandez-Serra, J. Chem. Phys. 134, 024516 (2011).

39 B. Kolb and T. Thonhauser, Phys. Rev. B 84, 045116 (2011).

40 O. Akin-Ojo and F. Wang, Chem. Phys. Lett. 513, 59 (2011).

41 B. Pamuk, J. M. Soler, R. Ramírez, C. P. Herrero, P. W. Stephens, P. B. Allen, and M. V. Fernández-Serra, Phys. Rev. Lett. 108, 193003 (2012).

42 M. J. Gillan, Phil. Mag. A 58, 257 (1988).

43 D. M. Ceperley, Rev. Mod. Phys. 67, 279 (1995).

44 R. Martonák, D. Donadio, and M. Parrinello, J. Chem. Phys. 122, 134501 (2005).

45 D. T. Bowron, J. L. Finney, A. Hallbrucker, I. Kohl, T. Loerting, E. Mayer, and A. K. Soper, J. Chem. Phys. 125, 194502 (2006).

46 T. Loerting, K. Winkel, M. Seidl, M. Bauer, C. Mitterdorfer, P. H. Handle, C. G. Salzmann, E. Mayer, J. L. Finney, and D. T. Bowron, Phys. Chem. Chem. Phys. 13, 8783 (2011).

47 S. Habershon, T. E. Markland, and D. E. Manolopoulos, J. Chem. Phys. 131, 024501 (2009).

48 S. Habershon and D. E. Manolopoulos, Phys. Chem. Chem. Phys. 13, 19714 (2011).

49 R. Ramírez and C. P. Herrero, Phys. Rev. B 84, 064130 (2011).

50 R. Ramírez and C. P. Herrero, J. Chem. Phys. 133, 144511 (2010).

51 C. P. Herrero and R. Ramírez, J. Chem. Phys. 134, 094510 (2011).

52 S. Habershon and D. E. Manolopoulos, J. Chem. Phys. 135, 224111 (2011).

53 J. Urquidi, C. J. Benmore, J. Neuefeind, B. Tomberli, C. A. Tulk, M. Guthrie, P. A. Egelstaff, and D. D. Klug, J. Phys.: Condens. Matter 15, 3657 (2003).

54 T. E. Markland, J. A. Morrone, B. J. Berne, K. Miyazaki, E. Rabani, and D. R. Reichman, Nature Phys. 7, 134 (2011).

55 R. P. Feynman, Statistical Mechanics (Addison-Wesley, New York, 1972).

56 H. Kleinert, Path Integrals in Quantum Mechanics, Statistics and Polymer Physics (World Scientific, Singapore, 1990). 
57 G. J. Martyna, M. E. Tuckerman, D. J. Tobias, and M. L. Klein, Mol. Phys. 87, 1117 (1996).

58 M. E. Tuckerman and A. Hughes, in Classical and Quantum Dynamics in Condensed Phase Simulations, edited by B. J. Berne, G. Ciccotti, and D. F. Coker (Word Scientific, Singapore, 1998), p. 311.

59 M. E. Tuckerman, in Quantum Simulations of Complex Many-Body Systems: From Theory to Algorithms, edited by J. Grotendorst, D. Marx, and A. Muramatsu (NIC, FZ Jülich, 2002), p. 269.

60 V. Buch, P. Sandler, and J. Sadlej, J. Phys. Chem. B 102, 8641 (1998).

61 B. S. Gonzalez, E. G. Noya, C. Vega, and L. M. Sese, J. Phys. Chem. B 114, 2484 (2010).

${ }^{62}$ L. Hernández de la Peña, M. S. Gulam Razul, and P. G. Kusalik, J. Chem. Phys. 123, 144506 (2005).

63 T. F. Miller and D. E. Manolopoulos, J. Chem. Phys. 123, 154504 (2005).

${ }^{64}$ L. Hernández de la Peña and P. G. Kusalik, J. Chem. Phys. 125, 054512 (2006)

65 J.-Y. Chen and C.-S. Yoo, Proc. Nat. Acad. Sci. USA 108, 7685 (2011).

66 R. Martonák, D. Donadio, and M. Parrinello, Phys. Rev. Lett. 92, 225702 (2004).

67 C. P. Herrero and R. Ramirez, Phys. Rev. B 84, 224112 (2011).

68 D. Chandler, Introduction to modern statistical mechanics (Oxford University Press, Oxford, 1987).

69 G. P. Johari and O. Andersson, Phys. Rev. B 76, 134103 (2007).

70 E. Libowitzky, Monatshefte für Chemie 130, 1047 (1999).

${ }^{71}$ K. Nygård, M. Hakala, S. Manninen, A. Andrejczuk,
M. Itou, Y. Sakurai, L. G. M. Pettersson, and K. Hämäläinen, Phys. Rev. E 74, 031503 (2006).

72 C. G. Salzmann, T. Loerting, S. Klotz, P. W. Mirwald, A. Hallbrucker, and E. Mayer, Phys. Chem. Chem. Phys. 8, 386 (2006).

73 E. Whalley, Can. J. Chem. 55, 3429 (1977).

74 L. Ojamäe and K. Hermansson, J. Chem. Phys. 96, 9035 (1992).

75 M. C. Bellissent-Funel, J. Teixeira, and L. Bosio, J. Chem. Phys. 87, 2231 (1987).

76 J. L. Finney, D. T. Bowron, A. K. Soper, T. Loerting, E. Mayer, and A. Hallbrucker, Phys. Rev. Lett. 89, 205503 (2002).

77 J. L. Finney, A. Hallbrucker, I. Kohl, A. K. Soper, and D. T. Bowron, Phys. Rev. Lett. 88, 225503 (2002).

78 L. Bosio, G. P. Johari, and J. Teixeira, Phys. Rev. Lett. 56, 460 (1986).

79 M. F. Herman, E. J. Bruskin, and B. J. Berne, J. Chem. Phys. 76, 5150 (1982).

80 R. Ramírez, N. Neuerburg, M. V. Fernández-Serra, and C. P. Herrero, J. Chem. Phys. 137, 044502 (2012).

81 L. D. Landau and E. M. Lifshitz, Statistical Physics (Pergamon, Oxford, 1980), 3rd ed.

82 C. P. Herrero, J. Phys.: Condens. Matter 20, 295230 (2008).

83 C. P. Herrero and R. Ramírez, Phys. Rev. B 63, 024103 (2000).

${ }^{84}$ F. Sciortino, U. Essmann, H. E. Stanley, M. Hemmati, J. Shao, G. H. Wolf, and C. A. Angell, Phys. Rev. E 52, 6484 (1995).

85 J. S. Tse and M. L. Klein, Phys. Rev. Lett. 58, 1672 (1987).

86 J. S. Tse and M. L. Klein, J. Chem. Phys. 92, 3992 (1990). 